\title{
Structural Studies of Respirasome by Cryo-Electron Microscopy
}

\author{
Tae Jin Jeon, Ho Min Kim ${ }^{1}$, Seong Eon Ryu* \\ Department of Bioengineering, College of Engineering, Hanyang University, Seoul 04763, Korea \\ ${ }^{1}$ Graduate School of Medical Science \& Engineering, Korea Advanced Institute of Science and Technology (KAIST), \\ Daejeon 34141, Korea
}

*Correspondence to:

Ryu $S E$,

(iD) http://orcid.org/0000-0003-3335-326X

Tel: $+82-2-2220-4020$

Fax: +82-2-2220-4023

E-mail: ryuse@hanyang.ac.kr

Received October 25, 2018

Revised November 21, 2018

Accepted November 22, 2018
The respiratory chain complex forms a supercomplex (SC) in the inner mitochondrial membrane. This complex facilitates the process of electron transfer to produce the proton gradient used to synthesize ATP. Understanding the precise structure of the SC is considered an important challenge. However, it has not yet been reported. The development of a Cryo-electron microscopy (EM) technique provides an effective way to obtain high-resolution micrographs to determine the high-resolution three-dimensional structure of biomolecules. In this brief review, the currently reported Cryo-EM structures of the mammalian respirasome have been described in order to establish a direction for further research in the respiratory system.

Key Words: Cryo-electron microscopy, Biomolecule structure, Respiratory chain complex, Respirasome
All living organisms contain huge complex proteins called respirasomes. Their functions not only include the generation of ATP but also the regulation of many other cellular processes. The conversion of energy from nutrients into ATP is mainly carried out by the oxidative phosphorylation system (OXPHOS) within the inner mitochondrial membrane. These are located on the inner membrane and organized by five large enzymes, namely, complex I (NADH-ubiquinone oxidoreductase, CI), complex II (succinate-quinone oxidoreductase, CII), complex III (cytochrome bcl complex, CIII), complex IV (cytochrome c oxidase, CIV), and complex V (ATP synthase, CV) (Fowler \& Richardson, 1963; Hatefi, 1985). All the complexes, CI to CIV, play a role in oxidoreductase but $\mathrm{CV}$ alone synthesizes ATP from ADT using the proton gradient energy produced by the electron transfer from CI to CIV (Hatefi, 1985; Sazanov, 2015).

It has been reported that the formation of the SC may reduce the production of reactive oxygen species (ROS) during the electron transfer process (Lopez-Fabuel et al., 2016; Maranzana et al., 2013). Additionally, it can increase the efficiency of the electron transfer of mobile electron carriers such as ubiquinone (Q) and cytochrome c (cyt c) (Bianchi et al.,
2004). However, the precise functional and structural role of the SC has not yet been determined. The earlier Cryo-electron microscopy (EM) structures of SCs revealed that there were three minor complexes that comprise the F-shaped SC, i.e., $\mathrm{I}_{1}+\mathrm{III}_{2}+\mathrm{IV}_{1}$. However, these representations did not allow an understanding of certain interactions between individual complexes because of the architectural level of the EM density (Althoff et al., 2011; Dudkina et al., 2011). The current Cryo-EM structures provide evidence that the SCs are mainly formed via specific interactions through conserved subunits (Letts et al., 2016; Sousa et al., 2016; Wu et al., 2016). The Cryo-EM structure of SCs from four different species propose an electron transfer mechanism based on the classical Q-cycle model, which is still controversial, or an alternative model (Guo et al., 2017; Letts \& Sazanov, 2017; Letts et al., 2016; Sousa et al., 2016; Wu et al., 2016). This review highlights the major advances in our understanding of the SC structure by Cryo-EM and discusses what was learnt from the Cryo-EM structures obtained in recent studies with a focus on respiratory systems.

In most organisms, the CI, CIII, and CIV consist of closely associated macromolecules called supercomplexes (SCs), which

@ This is an open-access article distributed under the terms of the Creative Commons Attribution Non-Commercial License (http://creativecommons.org/licenses/by-nc/4.0) which permits unrestricted noncommercial use, distribution, and reproduction in any medium, provided the original work is properly cited.

Copyrights (c) 2018 by Korean Society of Microscopy 
facilitate cellular respiration (Althoff et al., 2011; Dudkina et al., 2008, 2011). Other studies have suggested that CI and CIII are preferentially associated, whereas the other respiratory complexes (CII and CIV) function independently (Bianchi et al., 2004). The current EM studies of eukaryotic repirasomes (Table 1) show that the three minor complexes comprise the F-shaped SC, i.e., $\mathrm{I}_{1}+\mathrm{III}_{2}+\mathrm{IV}_{1}$ (Althoff et al., 2011; Gu et al., 2016; Guo et al., 2017; Letts et al., 2016; Sousa et al., 2016; Wu et al., 2016). The first part is an L-shaped segment corresponding to $\mathrm{CI}$ while the other two spherical segments corresponding to CIII and CIV are located nearby (Fig. 1A). The dimensions of the SC are $300 \AA$ in length and $190 \AA$ in height (Fig. 1A). It has been considered that the NDUFA11 subunit of CI may contribute to the formation of a SC with the CIII dimer through direct side-chain interactions or through phospholipid molecules such as cardiolipin in the space between CI and CIII (Dudkina et al., 2011; Wu et al., 2016). These results suggest that a specific conformation of SC is necessary to facilitate the mitochondrial electron transport process. Moreover, Cryo-EM structures from bovine, ovine, and porcine samples have reveal that the SC structure can be divided into different states during 3D classification (Letts et al., 2016;

Table 1. Structures of respiratory supercomplex

\begin{tabular}{|c|c|c|c|c|c|}
\hline PDB code & State & Species & Method & Resolution ( & Author \\
\hline $2 \mathrm{YBB}$ & $\mathrm{I}_{1} \mathrm{III}_{2} \mathrm{IV}_{1}$ & Bovine & EM & 19.0 & (Althoff et al., 2011) \\
\hline $5 \mathrm{~J} 4 \mathrm{Z}$ & $\mathrm{I}_{1} \mathrm{III}_{2} \mathrm{IV}_{1}$ (tight) & Ovine & $\mathrm{EM}$ & 5.8 & (Letts et al., 2016) \\
\hline $5 \mathrm{~J} 7 \mathrm{Y}$ & $\mathrm{I}_{1} \mathrm{III}_{2} \mathrm{IV}_{1}$ (loose) & $"$ & $"$ & 6.7 & $"$ \\
\hline $5 \mathrm{GPN}$ & $\mathrm{I}_{1} \mathrm{III}_{2} \mathrm{IV}_{1}$ & Porcine & $\mathrm{EM}$ & 5.4 & (Gu et al., 2016) \\
\hline 5GUP & $\mathrm{I}_{1} \mathrm{III}_{2} \mathrm{IV}_{1}$ & Porcine & EM & 4.0 & (Wu et al., 2016) \\
\hline $5 \mathrm{LUF}$ & $\mathrm{I}_{1} \mathrm{III}_{2} \mathrm{IV}_{1}$ & Bovine & $\mathrm{EM}$ & 9.1 & (Sousa et al., 2016) \\
\hline $5 \mathrm{XTH}$ & $\mathrm{I}_{1} \mathrm{III}_{2} \mathrm{IV}_{1}$ & Human & $\mathrm{EM}$ & 3.9 & (Guo et al., 2017) \\
\hline $5 X T I$ & $\mathrm{I}_{2} \mathrm{III}_{2} \mathrm{IV}_{2}$ & $"$ & $"$ & 17.4 & $"$ \\
\hline
\end{tabular}

EM, electron microscopy.
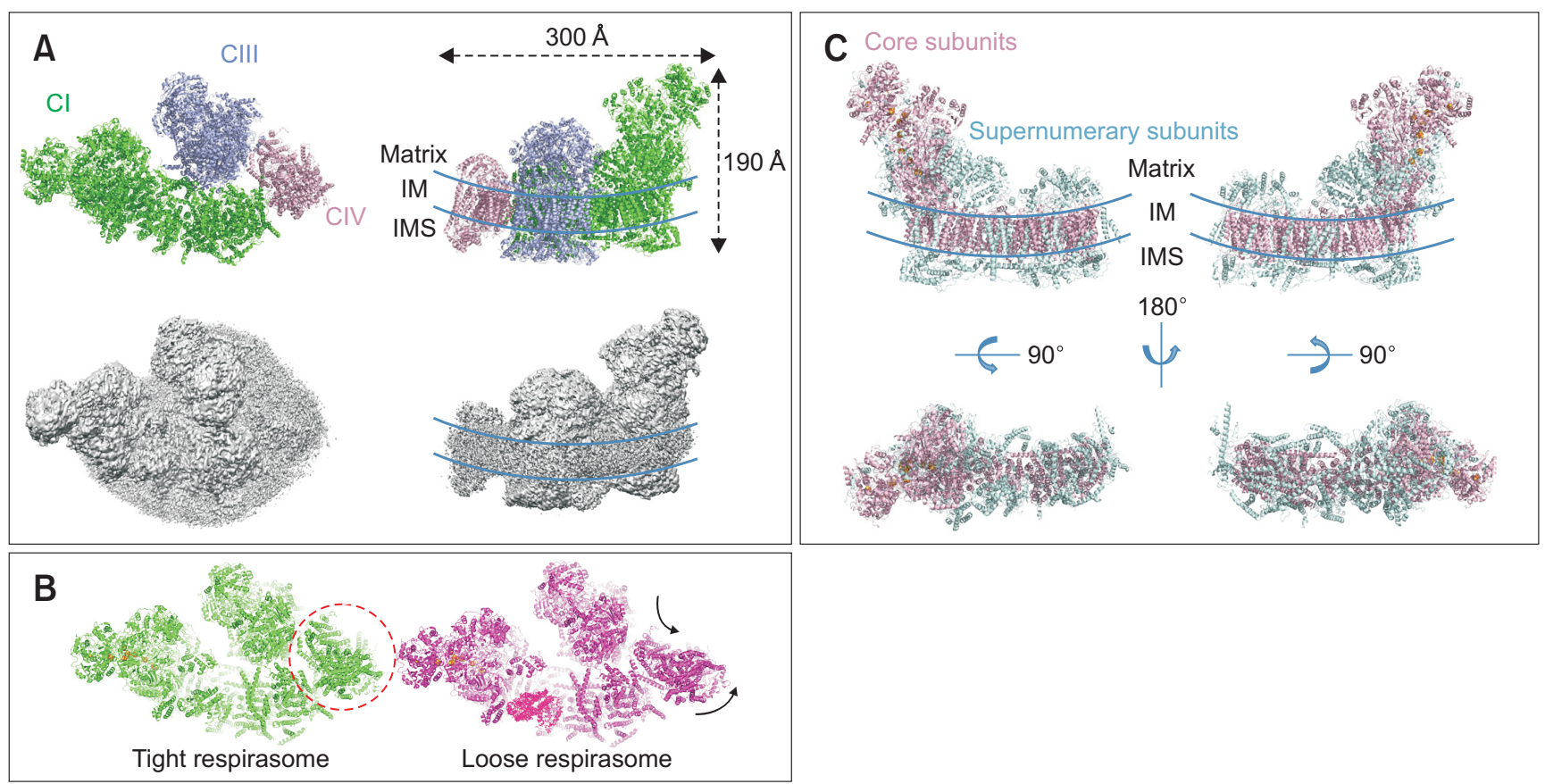

Fig. 1. Cryo-electron microscopy (EM) structure of mammalian supercomplex (SC) $\mathrm{I}_{1} \mathrm{III}_{2} \mathrm{IV}_{1}$ and CI. (A) The Cryo-EM structure of the porcine $\mathrm{SC} \mathrm{I}_{1} \mathrm{III}_{2} \mathrm{IV} \mathrm{V}_{1}$ (upper row, PDB code 5GUP) and the EM density map (bottom row, EMD-9539) (Wu et al., 2016). The map corresponding to porcine SC was generated from the 4.0 Å map at a contour level of 0.0412 using the UCSF chimera (Pettersen et al., 2004). Top views are from the matrix (left) and side views along the membrane (right). CI, CIII, and CIV are colored in green, light blue, and light pink, respectively. The transmembrane region is indicated by two curved lines. IM, inner membrane; IMS, intermembrane space; CI, complex I; CIII, complex III; CIV, complex IV. (B) Cartoon representation of tight (left, PDB code 5J4Z) and loose SC (right, PDB code 5J7Y) from ovine samples are shown viewed from the top view (Letts et al., 2016). CIV is shown in the red dashed circle. (C) Cartoon representation of the 3.9 Å resolution structure of the ovine CI (PDB code 5LNK). The core and extra subunits are colored (Fiedorczuk et al., 2016). The 14 core-subunits and 31 supernumerary subunits are colored in light magenta and cyan, respectively. 
Sousa et al., 2016; Wu et al., 2016). In ovine SCs, the two different states of SC show distinct conformational flexibility at CIV (Fig. 1B), which are referred to as the 'tight' or 'loose' respirasomes, indicating independent structural entities or different stages of assembly or disassembly (Letts et al., 2016). On the other hand, Cryo-EM studies of bovine and porcine SCs reveal that the matrix arm of CI and the overall structure of CIII show significant conformational changes (Sousa et al., 2016; Wu et al., 2016). In porcine SCs, one state shows that the matrix arms of CI and CIII move closer together to form a tighter SC than the other state. The minor class of bovine SC (class 2) revealed a rotational change of CIII based on the central axis of dimeric CIII compared to the major class SC (class 1) (Sousa et al., 2016). These results suggest that the large conformational changes of CIII and the matrix arm in CI strongly suggest an evidence of different conformational states during the electron transfer process.

Known to be one of the largest proteins, the mitochondrial CI consists of almost 45 subunits in eukaryotes (Carroll et al., $2003,2006)$ and 14 subunits in bacteria (Walker, 1992). The Cryo-EM studies of this macromolecule shown in Table 2 have revealed the "L" shaped density map consisting of hydrophilic and hydrophobic domains contained in 14 core subunits (Baradaran et al., 2013) and an extra 30 supernumerary subunits (Fiedorczuk et al., 2016; Vinothkumar et al., 2014; Zickermann et al., 2015) (Fig. 1C). The role of the supernumerary subunits was suggested to be one of protection and stabilization since they envelope the core subunit (Angerer et al., 2011). However, some subunits were considered to have a functional role (Hirst et al., 2003). Recently, the mechanism of the two states (A form and D form) of CI was reported by x-ray and Cryo-EM studies (Agip et al., 2018; Blaza et al., 2018; Fiedorczuk et al., 2016; Parey et al., 2018; Zhu et al., 2016; Zickermann et al., 2015). The loop $\beta 1-\beta 2$ in $49 \mathrm{kDa}$ and TMH1-2 in the ND3 subunit make a cavity during electron transfer which causes conformational changes (Wirth et al.,
2016; Zhu et al., 2016). These two states were also revealed in certain disease such as ischemia-reperfusion which cause rapid ROS production. In addition, it is known to be protected by the S-nitrosation of the cysteine switch (Chouchani et al., 2013). Other studies have reported that the $49 \mathrm{kDa}$ and PSST subunit make an inhibitor binding pocket (Darrouzet et al., 1998; Tocilescu et al., 2010) and points mutation of these subunits appear to associate with $\mathrm{CI}$ activity and the $\mathrm{IC}_{50}$ value of inhibitors such as rotenone, 2-decyl-4-quinazolinylamine (DQA), and $\mathrm{C}_{12} \mathrm{E}_{8}$ (Grgic et al., 2004; Kashani-Poor et al., 2001; Tocilescu et al., 2007). These inhibitors were regarded as therapeutic reagents (Ai et al., 2014; Alam \& Schmidt, 2004; Watabe \& Nakaki, 2008).

Most cells consume ATP for cell signaling, proliferation, and death. This energy is produced from proton pumping enzymes known as CI, CIII, and CIV (Bonora et al., 2012; Wallace, 2012). They facilitate intra-electron transfer by multiple redox cofactors. On the other hand, complex to complex electron transport is induced by carrier proteins such as ubiquinone and cytochrome c (Sarewicz \& Osyczka, 2015; Sazanov, 2015). This electron transport chain consists of an electron donor and acceptor that generate a proton gradient which is used as an energy source to produce ATP in CV (Jonckheere et al., 2012). The enzymatic assay analysis shows that the assembly of SC limits the production of ROS from CI (Maranzana et al., 2013). These results reveal that CI has to to be assembled with CIII, thus resulting in high mitochondrial respiration and low ROS production. The previous Q-cycle model assumes that the $\mathrm{Q}_{i}$ and $\mathrm{Q}_{\mathrm{o}}$ sites of CIII can play a role in the acceptance of ubiquinone and ubiquinol, respectively, and that only one electron of ubiquinol can be delivered to cytochrome c (Mitchell, 1975a, 1975b; Pietras et al., 2016). In order to transfer electrons to $\mathrm{cl}$, the iron-sulfur protein (ISP) has to move on a hinge by almost $15 \AA$ (Iwata et al., 1998). Additionally, recent studies have revealed that the active monomer of the dimeric CIII near the disordered ISP is

Table 2. Structures of respiratory complex I

\begin{tabular}{|c|c|c|c|c|c|}
\hline PDB code & State & Species & Method & Resolution ( $(\AA)$ & Author \\
\hline 3M9S & N/A & Thermus thermophilus & X-ray & 4.5 & (Efremov et al., 2010) \\
\hline 4HEA & N/A & T. thermophilus & X-ray & 3.3 & (Baradaran et al., 2013) \\
\hline 4UQ8 & N/A & Bovine & EM & 4.95 & (Vinothkumar et al., 2014) \\
\hline $4 \mathrm{WZ7}$ & Deactive & Yarrowia lipolytica & X-ray & 3.6 & (Zickermann et al., 2015) \\
\hline 5LC5 & Active & Bovine & EM & 4.35 & (Zhu et al., 2016) \\
\hline 5LDW & Deactive & $"$ & $"$ & 4.27 & $"$ \\
\hline $5 \mathrm{LDX}$ & N/A & $"$ & $"$ & 5.6 & $"$ \\
\hline 5LNK & Deactive & Ovine & EM & 3.9 & (Fiedorczuk et al., 2016) \\
\hline 5031 & Deactive & Bovine & $\mathrm{EM}$ & 4.13 & (Blaza et al., 2018) \\
\hline $6 \mathrm{G} 2 \mathrm{~J}$ & Active & Mouse & EM & 3.3 & (Agip et al., 2018) \\
\hline $6 \mathrm{G} 72$ & Deactive & $"$ & $"$ & 3.9 & " \\
\hline $6 \mathrm{GCS}$ & Deactive & Y. lipolytica & EM & 4.32 & (Parey et al., 2018) \\
\hline
\end{tabular}

N/A, not applicable; EM, electron microscopy. 

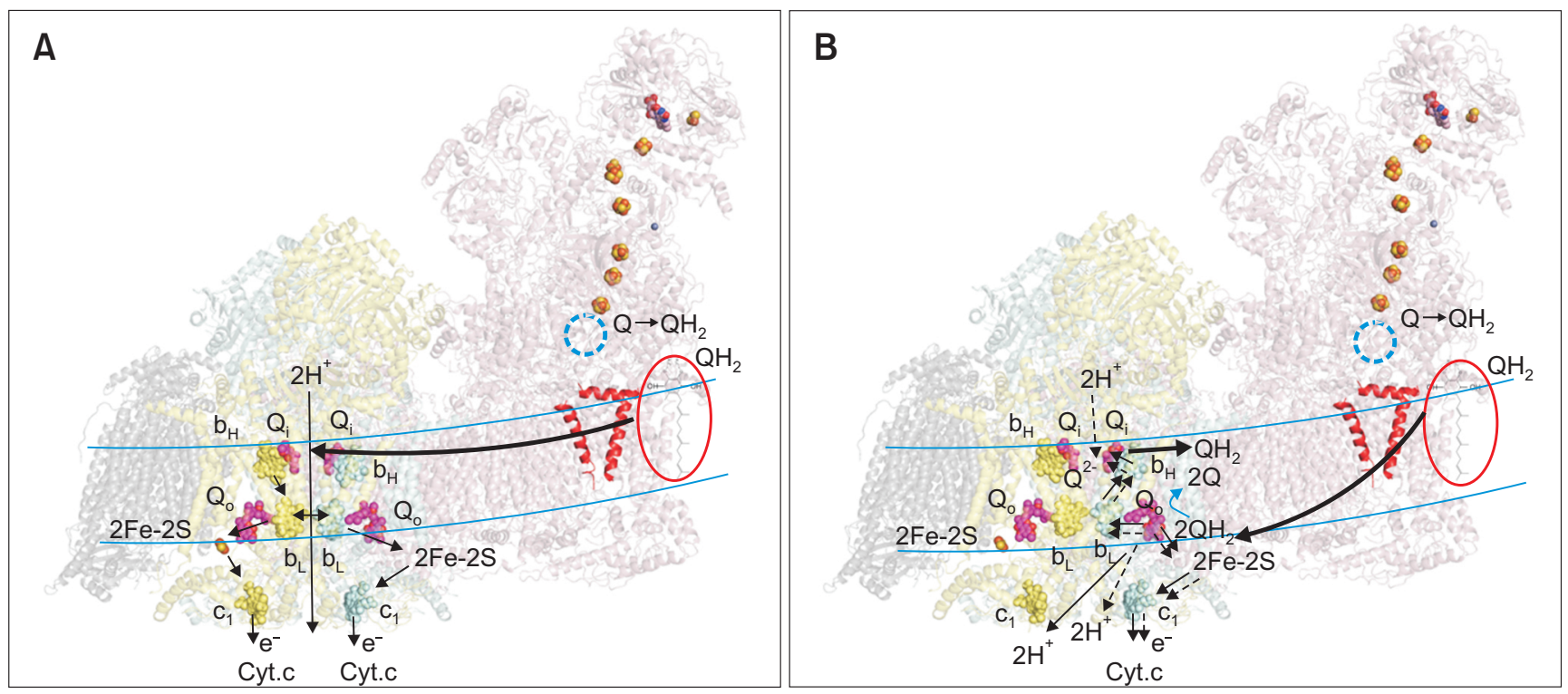

Fig. 2. Two proposed mechanisms of electron transfer. Symmetric (A) and asymmetric electron flow (B) through CIII are shown. Ubiquinol trail from CI to CIII is shown as a black thick line. The electron trail and transport of protons are shown as a black line. The hemes and FeS clusters are shown as spheres and the ubiquinol molecule are shown as sticks in the red oval. The ubiquinone binding site is shown in the blue dashed circle. The transmembrane region is indicated by two curved lines. The proximal and distal domains of CIII are colored in cyan and yellow, respectively. The CI and CIV are colored in magenta and grey, respectively. CI, complex I; CIII, complex III; CIV, complex IV.

surrounded by CI and CIV as well as the distal CIII monomer for asymmetric electron flow (Letts \& Sazanov, 2017; Letts et al., 2016; Sousa et al., 2016) (Fig. 2B). Interestingly, the human megacomplex revealed a $17 \AA$ resolution of circular architecture with an arrangement consisting of $\mathrm{I}_{2}+\mathrm{III}_{2}+\mathrm{IV}_{2}$ (Guo et al., 2017). Unlike the previous Q-cycle model, Guo et al. (2017) suggested that the ubiquinol released from CI can only bind to the $\mathrm{Q}_{\mathrm{i}}$ sites in the CIII dimer. Additionally, it can deliver two electrons to two cytochrome $\mathrm{c}$ at once for symmetric electron flow (Fig. 2A).

The structural studies of SC described by Cryo-EM at a resolution of 5 to $9 \AA$ did not allow the resolution of the biological mechanism at the atomic level. Although 40 years have passed since the Q-cycle model was proposed (Mitchell, 1975a, 1975b), a structure in which the native ubiquinone is bound to Q site has not yet reported (Gao et al., 2003). Thus far, it is difficult to explain how ubiquinol released from a CI can be inserted into the $\mathrm{Q}_{\mathrm{i}}$ or $\mathrm{Q}_{\mathrm{o}}$ site of CIII. Letts et al. (2016) demonstrate that there is no protein-mediated substrate channel connecting the Q binding sites of CI and CIII. However, metabolic control analysis revealed that the formation of SC increases the efficiency of electron transport through substrate channeling between CI and CIII (Bianchi et al., 2003, 2004). In addition, the diffusion issue of ubiquinol released from CI by which it can easily diffuse into the membrane during the electron transfer process may be accelerated without substrate channeling. However, the details remain controversial (Blaza et al., 2014; Enriquez, 2016; Letts et al., 2016; Wu et al.,
2016). An understanding of the precise mechanism of electron transfer from CI to CIII is important to verify the cause of ROS production. Thus, protein research at the molecular level is urgently required. Therefore, the atomic structure of the respirasome must be determined to understand protein interactions are mediated by lipids, cofactors, and ligands. Recently, innovative advances in Cryo-EM technology have revealed the possibilities of obtaining near atomic detail from giant membrane protein. However, unlike many structures resolved above $3 \AA$ resolution, many macromolecules including the respirasome still pose some difficulties in structural studies due to their flexibility. Hopefully, there will be many opportunities in the Cryo-EM field to facilitate research in the future, including more advanced technologies. For example, a section of cells analyzed by in situ structural biology involving a Cryo-electron tomography and sub-tomogram averaging may lead to the next revolution in macromolecular biology (Chung \& Jung, 2018; Chung \& Kim, 2017).

\section{CONFLICT OF INTEREST}

No potential conflict of interest relevant to this article was reported. 


\section{REFERENCES}

Agip A A, Blaza J N, Bridges H R, Viscomi C, Rawson S, Muench S P, and Hirst J (2018) Cryo-EM structures of complex I from mouse heart mitochondria in two biochemically defined states. Nat. Struct. Mol. Biol. 25, 548-556.

Ai Q, Jing Y, Jiang R, Lin L, Dai J, Che Q, Zhou D, Jia M, Wan J, and Zhang L (2014) Rotenone, a mitochondrial respiratory complex I inhibitor, ameliorates lipopolysaccharide/D-galactosamine-induced fulminant hepatitis in mice. Int. Immunopharmacol. 21, 200-207.

Alam M and Schmidt W J (2004) Mitochondrial complex I inhibition depletes plasma testosterone in the rotenone model of Parkinson's disease. Physiol. Behav. 83, 395-400.

Althoff T, Mills D J, Popot J L, and Kuhlbrandt W (2011) Arrangement of electron transport chain components in bovine mitochondrial supercomplex $I_{1} I_{2} I I_{1}$. EMBO J. 30, 4652-4664.

Angerer H, Zwicker K, Wumaier Z, Sokolova L, Heide H, Steger M, Kaiser S, Nubel E, Brutschy B, Radermacher M, Brandt T, and Zickermann $V$ (2011) A scaffold of accessory subunits links the peripheral arm and the distal proton-pumping module of mitochondrial complex I. Biochem. J. 437, 279-288.

Baradaran R, Berrisford J M, Minhas G S, and Sazanov L A (2013) Crystal structure of the entire respiratory complex I. Nature 494, 443-448.

Bianchi C, Fato R, Genova M L, Castelli G P, and Lenaz G (2003) Structural and functional organization of Complex I in the mitochondrial respiratory chain. BioFactors 18, 3-9.

Bianchi C, Genova M L, Castelli G P, and Lenaz G (2004) The mitochondrial respiratory chain is partially organized in a supercomplex assembly - Kinetic evidence using flux control analysis. J. Biol. Chem. 279, 36562-36569.

Blaza J N, Serreli R, Jones A J, Mohammed K, and Hirst J (2014) Kinetic evidence against partitioning of the ubiquinone pool and the catalytic relevance of respiratory-chain supercomplexes. Proc. Natl. Acad. Sci. USA 111, 15735-15740.

Blaza J N, Vinothkumar K R, and Hirst J (2018) Structure of the deactive state of mammalian respiratory complex I. Structure 26, 312-319.

Bonora M, Patergnani S, Rimessi A, De M E, Suski J M, Bononi A, Giorgi C, Marchi S, Missiroli S, Poletti F, Wieckowski M R, and Pinton P (2012) ATP synthesis and storage. Purinergic Signal 8, 343-357.

Carroll J, Fearnley I M, Shannon R J, Hirst J, and Walker J E (2003) Analysis of the subunit composition of complex I from bovine heart mitochondria. Mol. Cell. Proteomics 2, 117-126.

Carroll J, Fearnley I M, Skehel J M, Shannon R J, Hirst J, and Walker J E (2006) Bovine complex I is a complex of 45 different subunits. J. Biol. Chem. 281, 32724-32727.

Chouchani E T, Methner C, Nadtochiy S M, Logan A, Pell V R, Ding S, James A M, Cocheme H M, Reinhold J, Lilley K S, Partridge L, Fearnley I M, Robinson A J, Hartley R C, Smith R A, Krieg T, Brookes P S, and Murphy M P (2013) Cardioprotection by S-nitrosation of a cysteine switch on mitochondrial complex I. Nat. Med. 19, 753-759.

Chung J H and Kim H M (2017) The nobel prize in chemistry 2017: highresolution cryo-electron microscopy. Appl. Microsc. 47, 218-222.

Chung J M and Jung H S (2018) Cryo-electron tomography: a tool for in situ structural analysis of macromolecular complexes. Appl. Spectrosc. Rev. 53, 195-202.

Darrouzet E, Issartel J P, Lunardi J, and Dupuis A (1998) The 49-kDa sub- unit of NADH-ubiquinone oxidoreductase (Complex I) is involved in the binding of piericidin and rotenone, two quinone-related inhibitors. FEBS Lett. 431, 34-38.

Dudkina N V, Kudryashev M, Stahlberg H, and Boekema E J (2011) Interaction of complexes I, III, and IV within the bovine respirasome by single particle cryoelectron tomography. Proc. Natl. Acad. Sci. USA 108, 15196-15200.

Dudkina N V, Sunderhaus S, Boekema E J, and Braun H P (2008) The higher level of organization of the oxidative phosphorylation system: mitochondrial supercomplexes. J. Bioenerg. Biomembr. 40, 419-424.

Efremov R G, Baradaran R, and Sazanov L A (2010) The architecture of respiratory complex I. Nature $\mathbf{4 6 5}, 441-445$.

Enriquez J A (2016) Supramolecular organization of respiratory complexes. Annu. Rev. Physiol. 78, 533-561.

Fiedorczuk K, Letts J A, Degliesposti G, Kaszuba K, Skehel M, and Sazanov $L A$ (2016) Atomic structure of the entire mammalian mitochondrial complex I. Nature 538, 406-410.

Fowler L R, and Richardson S H (1963) Studies on the electron transfer system. J. Biol. Chem. 238, 456-463.

Gao X, Wen X, Esser L, Quinn B, Yu L, Yu C A, and Xia D (2003) Structural basis for the quinone reduction in the bc1 complex: a comparative analysis of crystal structures of mitochondrial cytochrome bc1 with bound substrate and inhibitors at the Qi site. Biochemistry 42, 90679080.

Grgic L, Zwicker K, Kashani-Poor N, Kerscher S, and Brandt U (2004) Functional significance of conserved histidines and arginines in the 49-kDa subunit of mitochondrial complex I. J. Biol. Chem. 279, 21193-21199.

Gu J, Wu M, Guo R, Yan K, Lei J, Gao N, and Yang M (2016) The architecture of the mammalian respirasome. Nature 537, 639-643.

Guo R, Zong S, Wu M, Gu J, and Yang M (2017) Architecture of human mitochondrial respiratory megacomplex $\mathrm{I}_{2} \mathrm{II}_{2} \mathrm{IV}_{2}$. Cell 170, 1247-1257.

Hatefi $Y$ (1985) The mitochondrial electron transport and oxidative phosphorylation system. Annu. Rev. Biochem. 54, 1015-1069.

Hirst J, Carroll J, Fearnley I M, Shannon R J, and Walker J E (2003) The nuclear encoded subunits of complex I from bovine heart mitochondria. Biochim. Biophys. Acta 1604, 135-150.

Iwata S, Lee J W, Okada K, Lee J K, Iwata M, Rasmussen B, Link T A, Ramaswamy S, and Jap B K (1998) Complete structure of the 11-subunit bovine mitochondrial cytochrome bc1 complex. Science $\mathbf{2 8 1}$, 64-71.

Jonckheere A I, Smeitink J A M, and Rodenburg R J T (2012) Mitochondrial ATP synthase: architecture, function and pathology. J. Inherit. Metab. Dis. 35, 211-225.

Kashani-Poor N, Zwicker K, Kerscher S, and Brandt U (2001) A central functional role for the 49-kDa subunit within the catalytic core of mitochondrial complex I. J. Biol. Chem. 276, 24082-24087.

Letts J A, Fiedorczuk K, and Sazanov L A (2016) The architecture of respiratory supercomplexes. Nature 537, 644-648.

Letts J A and Sazanov L A (2017) Clarifying the supercomplex: the higherorder organization of the mitochondrial electron transport chain. Nat. Struct. Mol. Biol. 24, 800-808.

Lopez-Fabuel I, Le Douce J, Logan A, James A M, Bonvento G, Murphy M 
P, Almeida A, and Bolanos J P (2016) Complex I assembly into supercomplexes determines differential mitochondrial ROS production in neurons and astrocytes. Proc. Natl. Acad. Sci. USA 113, 1306313068.

Maranzana E, Barbero G, Falasca A I, Lenaz G, and Genova M L (2013) Mitochondrial respiratory supercomplex association limits production of reactive oxygen species from complex I. Antioxid. Redox Signal. 19, 1469-1480.

Mitchell P (1975a) The protonmotive Q cycle: a general formulation. FEBS Lett. 59, 137-139.

Mitchell P (1975b) Protonmotive redox mechanism of the cytochrome b-c1 complex in the respiratory chain: protonmotive ubiquinone cycle. FEBS Lett. 56, 1-6.

Parey K, Brandt U, Xie H, Mills D J, Siegmund K, Vonck J, Kuhlbrandt W, and Zickermann V (2018) Cryo-EM structure of respiratory complex I at work. eLife 7, e39213.

Pettersen E F, Goddard T D, Huang C C, Couch G S, Greenblatt D M, Meng E C, and Ferrin T E (2004) UCSF Chimera-a visualization system for exploratory research and analysis. J. Comput. Chem. 25, 1605-1612.

Pietras R, Sarewicz M, and Osyczka A (2016) Distinct properties of semiquinone species detected at the ubiquinol oxidation $\mathrm{Q}(0)$ site of cytochrome bc(1) and their mechanistic implications. J. R. Soc. Interface 13, 20160133.

Sarewicz M and Osyczka A (2015) Electronic connection between the quinone and cytochrome $\mathrm{c}$ redox pools and its role in regulation of mitochondrial electron transport and redox signaling. Physiol. Rev. 95, 219-243.

Sazanov L A (2015) A giant molecular proton pump: structure and mechanism of respiratory complex I. Nat. Rev. Mol. Cell Biol. 16, 375-388.
Sousa J S, Mills D J, Vonck J, and Kuhlbrandt W (2016) Functional asymmetry and electron flow in the bovine respirasome. eLife 5, e21290.

Tocilescu M A, Fendel U, Zwicker K, Kerscher S, and Brandt U (2007) Exploring the ubiquinone binding cavity of respiratory complex I. J. Biol. Chem. 282, 29514-29520.

Tocilescu M A, Zickermann V, Zwicker K, and Brandt U (2010) Quinone binding and reduction by respiratory complex I. Biochim. Biophys. Acta 1797, 1883-1890.

Vinothkumar K R, Zhu J, and Hirst J (2014) Architecture of mammalian respiratory complex I. Nature $\mathbf{5 1 5}, 80-84$.

Walker J E (1992) The NADH:ubiquinone oxidoreductase (complex I) of respiratory chains. Q. Rev. Biophys. 25, 253-324.

Wallace D C (2012) Mitochondria and cancer. Nat. Rev. Cancer 12, 685698.

Watabe M and Nakaki T (2008) Mitochondrial complex I inhibitor rotenone inhibits and redistributes vesicular monoamine transporter 2 via nitration in human dopaminergic SH-SY5Y cells. Mol. Pharmacol. 74, 933-940.

Wirth C, Brandt U, Hunte C, and Zickermann V (2016) Structure and function of mitochondrial complex I. Biochim. Biophys. Acta 1857, 902914.

Wu M, Gu J, Guo R, Huang Y, and Yang M (2016) Structure of mammalian respiratory supercomplex $\mathrm{I}_{1} \mathrm{III}_{2} \mathrm{I} \mathrm{V}_{1}$. Cell 167, 1598-1609.

Zhu J, Vinothkumar K R, and Hirst J (2016) Structure of mammalian respiratory complex I. Nature 536, 354-358.

Zickermann V, Wirth C, Nasiri H, Siegmund K, Schwalbe H, Hunte C, and Brandt U (2015) Mechanistic insight from the crystal structure of mitochondrial complex I. Science $\mathbf{3 4 7}$, 44-49. 